\title{
PET/CT Image Denoising and Segmentation based on a Multi Observation and a Multi Scale Markov Tree Model
}

\author{
H.HANZOULI, Student Member IEEE, J.LAPUYADE-LAHORGUE, E.MONFRINI, G. DELSO, W. \\ PIECZYNSKI, D.VISVIKIS, Senior Member IEEE, M.HATT, Member IEEE
}

\begin{abstract}
This work deals with the use of a probabilistic quadtree graph (Hidden Markov Tree, HMT) to provide fast computation, improved robustness and an effective interpretational framework for image analysis and processing in oncology. Thanks to two efficient aspects (multi observation and multi resolution) of HMT and Bayesian inference, we exploited joint statistical dependencies between hidden states to handle the entire data stack. This new flexible framework was applied first to mono modal PET image denoising taking into consideration simultaneously the Wavelets and Contourlets transforms through multi observation capability of the model. Secondly, the developed approach was tested for multi modality image segmentation in order to take advantage of the high resolution of the morphological computed tomography (CT) image and the high contrast of the functional positron emission tomography (PET) image. On the one hand, denoising performed through the wavelet-contourlet combined multi observation HMT led to the best trade-off between denoising and quantitative bias compared to wavelet or contourlet only denoising. On the other hand, PET/CT segmentation led to a reliable tumor segmentation taking advantage of both PET and CT complementary information regarding tissues of interest. Future work will investigate the potential of the HMT for PET/MR and multi tracer PET image analysis. Moreover, we will investigate the added value of Pairwise Markov Tree (PMT) models and evidence theory within this context.
\end{abstract}

Manuscript received November 15, 2013. This work was supported by a $\mathrm{PhD}$ grant from the French Ministry of Research.

H. Hanzouli is with the LaTIM, INSERM UMR 1101, 29200 Brest, France (e-mail: hanzouli@univ-brest.fr).

J. Lapuyade-Lahorgue, is with the LaTIM, INSERM UMR 1101, 29200 Brest, France. (e-mail: jerome.lapuyade.lahorgue@gmail.com).

E. Monfrini, is with Telecom Sud-Paris, CITI department, CNRS, UMR 5157 SAMOVAR, 91011 Evry, France. (e-mail: emmanuel.monfrini@telecomsudparis.eu).

G. Delso is with Department of Medical Imaging, University Hospital, Rämistrasse 100, 8091, Zurich, Switzerland. (e-mail: gaspar.delso@usz.ch)

W. Pieczynski, is with Telecom Sud-Paris, CITI department, CNRS, UMR 5157 SAMOVAR, 91011 Evry, France. (e-mail: wojciech.pieczynski@telecomsudparis.eu).

D. Visvikis is with the LaTIM, INSERM UMR 1101, 29200 Brest, France . (email: dimitris@univ-brest.fr).

M. Hatt is with the LaTIM, INSERM UMR 1101, 29200 Brest, France. (e-mail: hatt@univ-brest.fr)
Keywords: Positron Emission Tomography (PET), Computed Tomography (CT), Bayesian inference, Hidden Markov Trees (HMT), Wavelet and Contourlet analysis

\section{INTRODUCTION}

$T$ he use of $3 \mathrm{D}$ image processing has increased in the last decade for medical applications. Clinicians have to navigate, view, analyze, segment, and interpret a growing number of multi dimensional and multi resolution images. Visual analysis of the whole image stack is subjective and time consuming owing to increasing data volumes as well as image characteristics' variability in terms of noise, contrast, texture and resolution. Automated analysis of all this multi modality information may thus potentially improve overall patient management.

Statistical approaches have been proven useful for the design of robust and efficient processing and analysis methods. Resorting to a Bayesian scheme based on Hidden Markov Models (HMM) is attractive when dealing with a large number of observations. These models owe their flexibility to their ability to simultaneously take into account inter- and intrascale spatial Markov statistical dependencies as well as in terms of estimating in a non-supervised manner model's parameters.

This work presents the development of a statistical multi resolution framework under a Markovian assumption, in order to address multi modality image analysis tasks. In this work the developed model was tested in Positron Emission Tomography/Computed Tomography (PET/CT) denoising and segmentation tasks.

\section{MATERIALS AND METHODS}

\section{A. STATISTICAL INFERENCE}

From a statistical point of view, the spatial dependencies between hidden random variables were first introduced as contextual priors. This introduction in statistical imaging makes each voxel labeling dependent on all of the others, which might lead to computational limitation issues. 
Nevertheless, when dealing with the Markovian assumption, this prior is usually formulated so that the conditional probability of a voxel label, given the labeling of all the other voxels, is reduced to the conditional probability given only the labeling of the neighboring voxels. Recently, it has been suggested to replace such purely spatial priors with Markov Tree priors, in which connections between variables are no longer handled only within the image voxels grid but also across scales. Thanks to its remarkable properties (multi observation and multi resolution joint statistics, as illustrated in Fig. 1) such a model (Hidden Markov Tree, HMT) [1] can provide fast computation, improved robustness and an effective interpretational process when handling the whole observed data simultaneously.

\section{A. HIDDEN MARKOV TREE (HMT)}

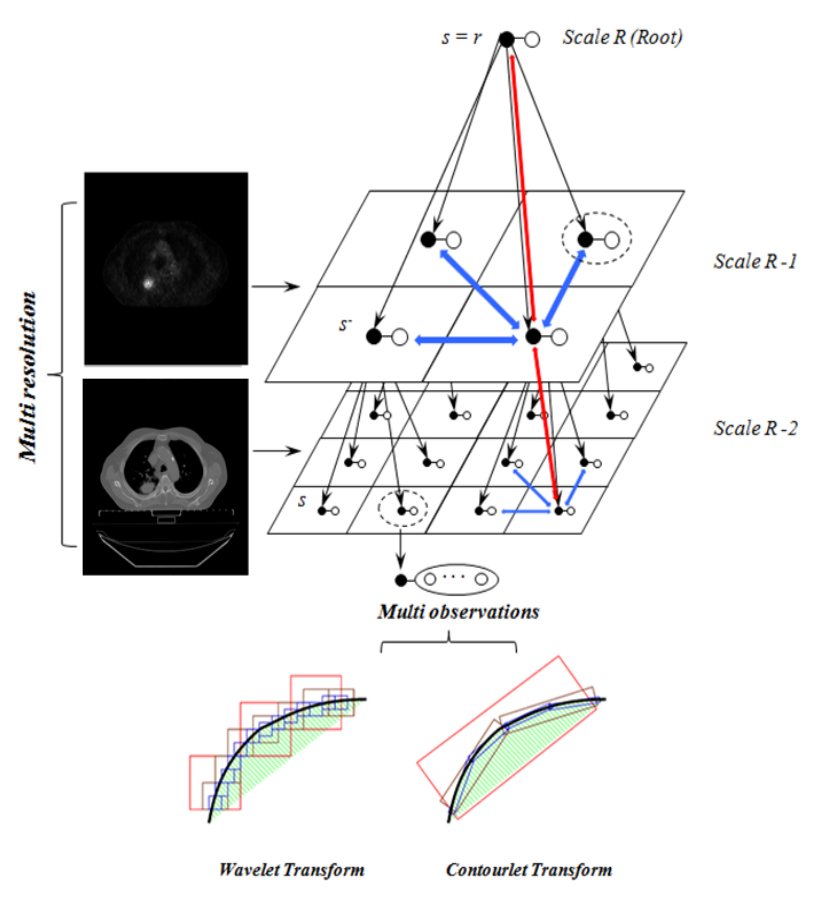

Fig. 1: Hidden Markov Tree Model

The HMT model is defined as follows: let $\mathrm{S}$ be a finite set of points and $\mathrm{X}=\left(\mathrm{X}_{\mathrm{s}}\right)_{\mathrm{s}} \mathrm{s}, \mathrm{Y}=\left(\mathrm{Y}_{\mathrm{s}}\right)_{\mathrm{s}} \mathrm{s}$ two stochastic processes indexed on $\mathrm{S}$. Each $\mathrm{X}_{\mathrm{s}}$ takes its values finite set of classes $\Omega=\left\{\omega_{1, \ldots} \omega_{\mathrm{k}}\right\}$ and $\mathrm{Y}_{\mathrm{s}}$ takes its values in the set of observations (real values). Let $\mathrm{S}^{1}, \ldots, \mathrm{S}^{\mathrm{R}}$ be a partition of $\mathrm{S}$ representing different "generations". Each $s \in S^{i}$ admits $s^{+} \in S^{i+1}$ (called his "children") in such a way that every element of $t \in S^{i+1}$ has a unique "parent" $t^{-} \in S^{i}$ such that $\left(s^{+}\right)^{-}=s$. We assume that $S^{1}$ is a singleton so as its element $\mathrm{S}_{\mathrm{r}}$ is called "root"). $\mathrm{X}$ is a Markov tree if:

$$
P(x)=P\left(x_{s_{r}}\right) \prod_{s \in S-S^{1}} P\left(x_{s} \mid x_{s^{-}}\right) .
$$

The likelihood of the observations $\mathrm{y}$ conditionally on $\mathrm{x}$ is expressed as the following product (assuming conditional independence):

$$
P(y \mid x)=\prod_{n=0}^{R} \prod_{s \epsilon S^{n}} P\left(y_{s} \mid x_{s}\right)
$$

The parameters of the HMT are estimated thanks to the iterative Stochastic Expectation Maximization (SEM) algorithm [2]. Modes of Posterior Marginal (MPM) [3] inference is performed using the marginal posteriors which are computed exactly by propagating information first from the "leaves" to the "root", and then in the inverse direction.

\section{APPLICATIONS}

\section{A. Wavelet-Contourlet based PET Denoising}

The developed framework was firstly evaluated for PET denoising. On the one hand, Wavelet based methods exploit observation-based transforms at different resolutions in order to better describe the whole observed information [4]. The major drawback of Wavelets is their limited ability in capturing directional information. On the other hand, the Contourlet transform [5] has emerged as an efficient tool able to capture the intrinsic geometrical structures such as smooth contours thanks to a high degree of directionality and anisotropy. This transform is however not optimal for isotropic structures, contrary to Wavelets.

Consequently, an approach combining both transforms may lead to optimal performance. In this work the multiobservation aspect of the proposed HMT was exploited in order to associate both Wavelets and Contourlets coefficients to each voxel (Fig.1), leading to a Wavelet-Contourlet Denoising (WCD). The proposed approach was carried out on realistic Monte Carlo simulations of the IEC phantom (a cylinder containing six spheres with diameters from 10 to 37 $\mathrm{mm}$, and filled with ${ }^{18} \mathrm{~F}$ ). Fig. 2 illustrates an example of WCD denoising results for one case $8: 1,4 \times 4 \times 4 \mathrm{~m}^{3}, 20$ million coincidences, compared with Wavelet Denoising (WD), Contourlet Denoising (CD) and the Gaussian filtering. Visual analysis of the difference results demonstrated that the boundaries of the cylinder and spheres were better preserved with $\mathrm{CD}$ and WCD than with WD that led to more loss of structural integrity. Mean $\pm \mathrm{SD}$ of measurements (Fig. 3) assessed through the different spheres' sizes demonstrated that a significant signal-to-noise ratio (SNR) increase was obtained with all the methods, however WCD was the one better preserving original intensities and contrast values.

Thus, the proposed approach (WCD) led to the best trade-off between SNR improvement and quantitative bias. 


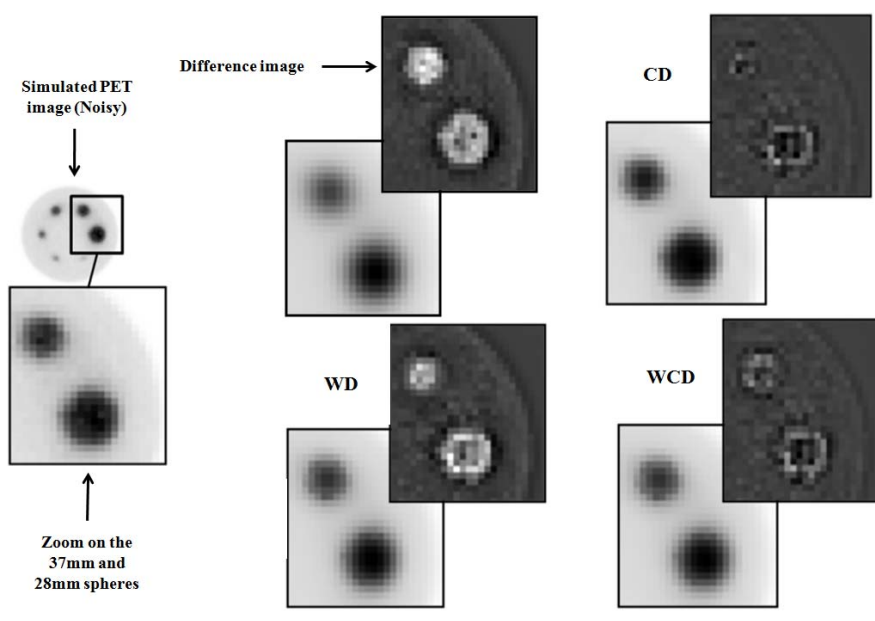

Fig. 2: Simulated image and difference images for Wavelet Denoising (WD), Contourlet Denoising (CD) and Wavelet-Contourlet denoising (WCD)

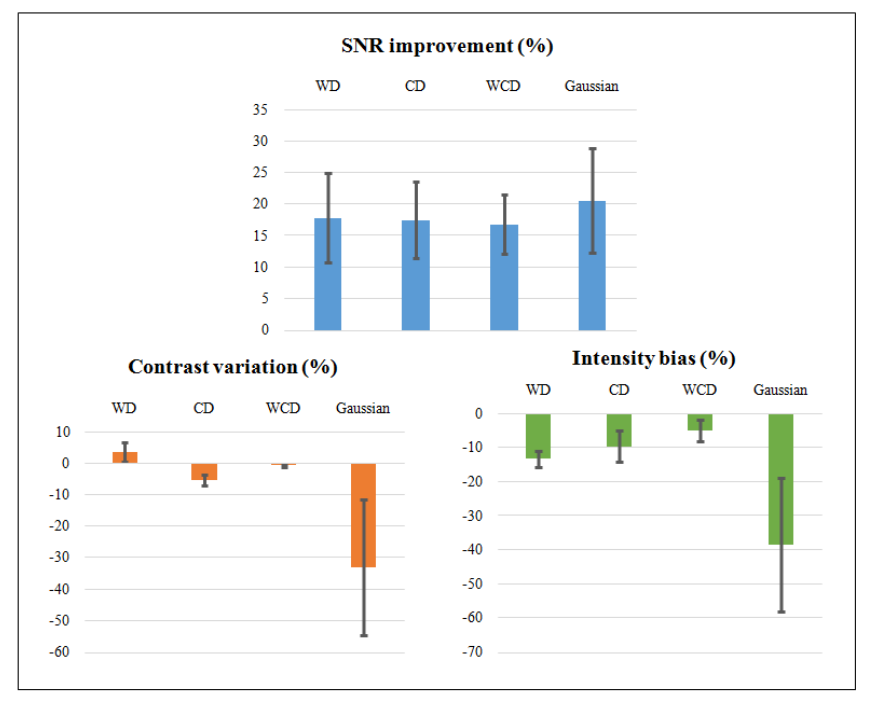

Fig. 3: SNR, contrast and intensity measurements assessed on the denoised simulated image with WD, CD, WCD and the Gaussian filtering

\section{B. Multi modal (PET/CT) image segmentation}

Image segmentation is an essential step in radiotherapy treatment planning. Clinicians manually delineate CT images fused with PET [6], in order to define the gross tumor volume (GTV). Manual delineation is a time consuming process prone to inter- and intra-observer variability. On the other hand, segmentation of lesions based on only one image modality when multiple complementary ones (PET, CT, MR) are available is also limited. In this work, we investigated the possibility to automate such a process by exploiting the HMT multi resolution framework to associate high resolution CT with lower resolution PET images (see Fig.1). The proposed segmentation results were carried out on three clinical multi modal dataset images (PET, CT). In the absence of realistic simulated CT images and other surrogate of truth such as histopathology volumes, the validation of the approach consisted in comparing HMT multimodal segmentation of the tumor with a statistical consensus of manual delineations performed by several radiation oncologists on fused PET/CT images. Results show a satisfactory agreement between the consensus of manual contours and our automated result (Fig. 4-5). As shown in TABLE I, WCD segmentation over the two datasets led to errors below $25 \%$ (table I).

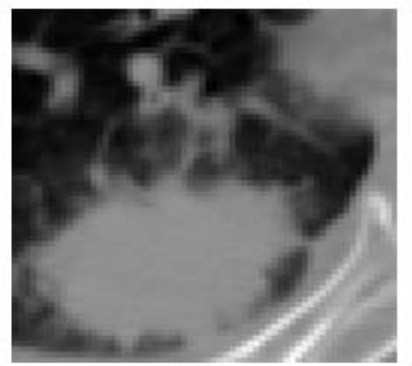

(a)

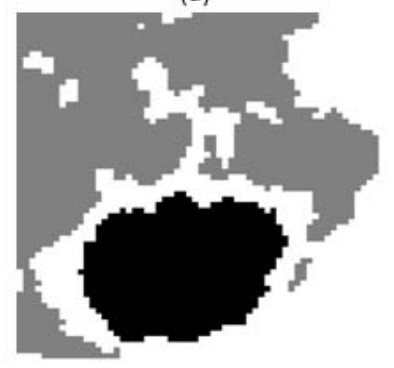

(c)

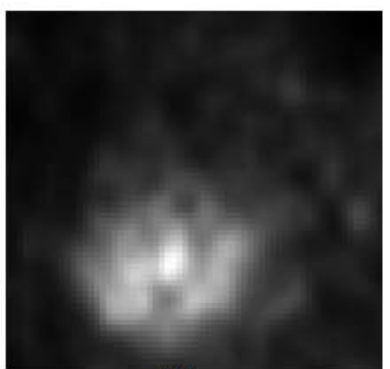

(b)

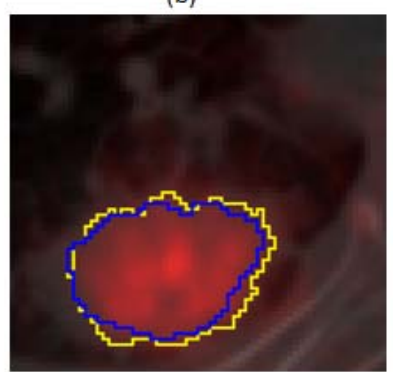

(d)
Fig. 4: First multi modal clinical dataset image: CT (a) and PET (b) cosegmented simultaneously (c) within the PET/CT HMT framework. (d) Overlaid manual reference (blue) and automated (yellow) contours on fused PET/CT images.

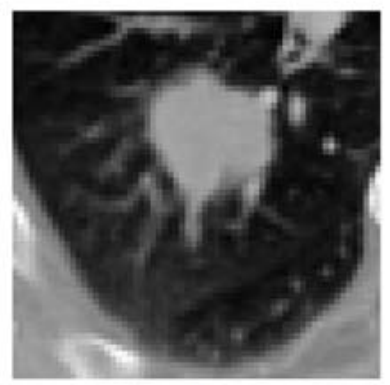

(a)

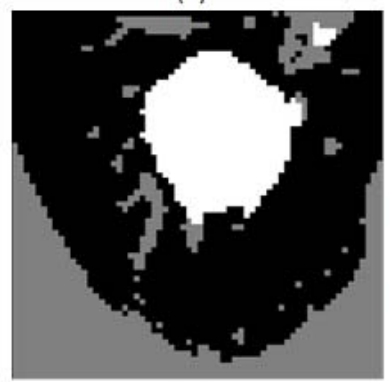

(c)

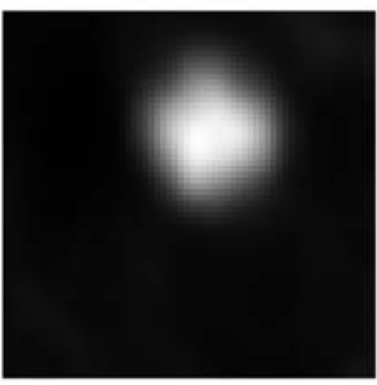

(b)

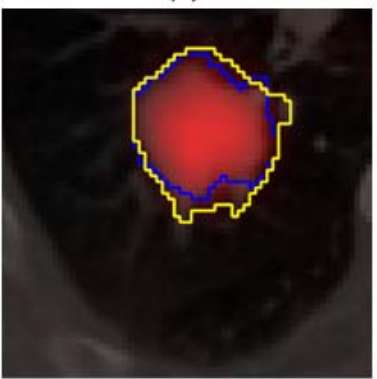

(d)
Fig. 5: Second multi modal clinical dataset image: CT (a) and PET(b) cosegmented simultaneously (c) within the PET/CT HMT framework. (d) Overlaid manual reference (blue) and automated (yellow) contours on fused $\mathrm{PET} / \mathrm{CT}$ images. 
TABLEI. FALSE NEGATIVE (FN) AND FALSE POSITIVE (FP) ERRORS MEASUREMENTS

\begin{tabular}{lll} 
& False Negative & False Positive \\
\hline First Dataset & $1,03 \%$ & $16,73 \%$ \\
Second Dataset & $1,56 \%$ & $20,03 \%$ \\
\hline
\end{tabular}

\section{CONCLUSION AND FURTHER WORK}

In this work, we developed a flexible framework based on HMT for multi modal image processing and analysis and demonstrated its exploitation for two different image processing applications, namely mono modal PET image denoising through the combination of two different spatiofrequential transforms, and multimodal PET/CT image tumor delineation. PET image denoising was performed within the HMT by exploiting its multi observation property, allowing the consideration of both Wavelets and Contourlets simultaneously in the model, with improved results relative to wavelet or contourlet denoising only. Multi modality tumor segmentation was performed by exploiting the hierarchical property of the HMT, allowing associating the high resolution CT image at the leafs of the tree, and the lower resolution PET image at the next higher scale in the tree. Future work will focus on exploiting this HMT model for PET/MR and PET multi tracer information, in addition to the use of Pairwise Markov Tree (PMT) [1] combined with evidence theory [7]. Validation of the PET/CT segmentation on datasets with histopathological reference will be also presented.

\section{REFERENCES}

[1] E. Monfrini, J.Lecompte, F. Desbouvries and W. Pieczynski. Image and signal restoration using pairwise Markov Trees. IEEE Workshop on Stat.Sign. Proc. 2003.

[2] G. Celeux, J. Diebolt. A stochastic approximation type EM algorithm for the mixture problem. Stochastics and Stochastics Reports, 41:127-146 (1992).

[3] P. Gonçalvès,J.-B. Durand. Statistical inference for hidden Markov tree models and application to wavelet tree. Technical report 4248, INRIA, Septembre 2001.

[4] P. Dutilleux. An implementation of the "algorithme à trous" to compute the wavelet transform. Time-Frequency Methods and Phase Space 1:298-304 (1989).

[5] D. D.-Y Po, M. N Do. Directional multiscale modeling of images using the contourlet transform. IEEE Trans. Im. Proc. 15(6):1610-20 (2006).

[6] PH. Jarritt, K. Carson, D. Visvikis. The role of PET/CT scanning in radiotherapy treatment planning. Br J Radiol, 79,27-35 (2006).

[7] P.Lanchantin and W. Pieczynski. Unsupervised Restoration of Hidden Nonstationary Markov Chains Using Evidential Priors. IEEE Transactions on Signal Processing, VOL. 53, NO. 8, August 2005. 